\title{
Hydroxyapatite-based Electrodes for Metal Detection in Wastewater
}

Mirko Magni ${ }^{a}$, Davide Sironi ${ }^{a}$, Michele Ferri ${ }^{b}$, Sebastiano Campisi ${ }^{b}$, Antonella Gervasini ${ }^{b}$, Pierangela Cristianic, Maddalena Papacchini ${ }^{\mathrm{d}}$, Stefano Trasatti ${ }^{\mathrm{a}}$

a Dipartimento di Scienze e Tecnologie Ambientali, Università degli Studi di Milano, via Golgi 19, 20133 Milano (Italy)

b Dipartimento di Chimica, Università degli Studi di Milano, via Golgi 19, 20133 Milano (Italy)

c RSE - Ricerca sul Sistema Energetico S.p.A., via Rubattino 54, 20134 Milano (Italy)

d INAIL Dipartimento Innovazioni Tecnologiche e Sicurezza degli Impianti, Prodotti e Insediamenti Antropici, Area di Ricerca Monteporzio Catone, Via di Fontana Candida 1, 00040 Monteporzio Catone, Roma (Italy)

Hydroxyapatite (HAp) is a biocompatible versatile material of formula $\mathrm{Ca}_{10}\left(\mathrm{PO}_{4}\right)_{6}(\mathrm{OH})_{2}$, insoluble in water within a wide $\mathrm{pH}$ range, chemically stable, relatively cheap and largely available. This mineral calcium phosphate has caught attention of scientists working in different fields of applied science, from medical engineering [1], to catalysis [2] and pollution remediation [3],[4]. For environmental application, the absorbent nature of HAp is, probably, the most valuable feature. In particular, heavy metal retention ability is attributed to ion-exchange ( $\mathrm{Ca}^{2+} /$ metal ion), surface adsorption/complexation, dissolution-precipitation mechanism, with single or combined action depending on the metal nature [5]. Combining intrinsic affinities of HAp for metals with ductility of electrochemistry is a valuable route to develop monitoring systems and/or pollution remediation protocols [6]. In doing so, the main obstacle for the exploitation of HAp as electrode materials is its electrical insulation nature. To overcome this limitation combination with conductive substrate is necessary, preparing either blends or composite materials.

In this context, a series of carbon-containing hydroxyapatite composites (C-HAp) have been prepared by coprecipitation synthesis, by varying the conductive carbon source. The prepared materials have been characterized by various physical-chemical techniques (FT-IR spectroscopy, XRPD, TEM-EDX, $\mathrm{N}_{2}$ adsorption/desorption analyses) and the electrical conductivity has been determined as a function of the carbon source. The most promising C-HAp composites have been used as electrode substrates to quantify some of the common heavy metals found in waste water from urban and/or industrial sites (e.g., $\mathrm{Pb}^{2+}, \mathrm{Cd}^{2+}$, $\mathrm{Cu}^{2+}, \mathrm{Zn}^{2+}$ ) using cyclic and differential voltammetry techniques. The sensitivity of C-HAp electrodes was compared with that of glassy carbon ones, chosen as reference material. Different electrode geometries have been taken into consideration (C-HAp powder pressed into a cavity electrode, or free-standing C-HAp one).

The work is still in progress and among possible alternative routes we are going to prepare directly HApbased electrodes by in situ electrodeposition of calcium phosphate on low cost electron collectors such as steel. The final aim is to employ the HAp-based electrode as cathode in microbial fuel cells that could act as sensors for the on-line detection of metal traces in the treated wastes.

References:

[1] J. Kolmas, S. Krukowski, A. Laskus, M. Jurkitewicz, Ceramics International, 2016, 42, 2472-2487.

[2] A. Fihri, C. Len, R. S. Varma, A. Solhy, Coordin. Chem. Rev. 2017, 347, 48-76.

[3] J. Reichert, J. G. P. Binner, J. Mater. Sci., 1996, 31, 1231-1241.

[4] A. Corami, S. Mignardi, V. Ferrini, J. Hazard. Mater., 2007, 146, 164-170.

[5] S. Campisi, C. Castellano, G. Gervasini, New J. Chem., 2018, 42, 4520-4530.

[6] M.A. El Mhammedi, M. Achak, R. Najih, M. Bakasse, A. Chtaini, Mater. Chem. Phys., 2009, 115, 567-571. 